\title{
CICLO DIÁRIO DE PRECIPITAÇÕES PLUVIAIS EM INTERVALOS DE CINCO MINUTOS NO MUNICÍPIO DE FORTALEZA
}

\author{
FRANCISCO EDSON PINHEIRO PESSOA, JOSÉ NILSON B. CAMPOS
}

\author{
Universidade Federal do Ceará, Centro de Tecnologia, Departamento de Engenharia Hidráulica e Ambiental, \\ Fortaleza, CE, Brasil \\ epessoa2010@yahoo.com.br,jnbcampos@gmail.com
}

Recebido Agosto de 2014 - Aceito Setembro de 2014

\begin{abstract}
RESUMO
O artigo estuda o ciclo diário de precipitações pluviais no município de Fortaleza, Ceará. Utilizou-se uma série de 30 anos de dados em intervalos de cinco minutos. Os dados são oriundos de registros pluviográficos da estação meteorológica da Universidade Federal do Ceará, no Campus do Pici. Para se obter a curva do ciclo diário, utilizou-se a análise de regressão não linear. Pesquisou-se o ajustamento dos dados a dois tipos de curvas: polinomial e senoidal. O ajustamento a um polinômio de terceiro grau resultou em uma curva de formato aproximadamente senoidal, com coeficiente de determinação $\mathrm{r}^{2}=0,9118$. Entretanto, a curva ajustada exibiu descontinuidade entre os valores das ordenadas no início e fim do ciclo diário. Desenvolveu-se um polinômio, denominado polinômio cíclico, que retira a descontinuidade nos extremos do ciclo diário. Obteve-se uma curva de forma nitidamente senoidal, com $r^{2}=0,8798$. Partiu-se, assim, para o ajustamento de funções do tipo senoidal. Foi testada a senoide simples e uma associação seno-cosseno. Com a combinação de senos e cossenos, chegou-se a uma curva com $\mathrm{r}^{2}=0,9113$, que foi considerada como a melhor representação do ciclo diário de chuvas em Fortaleza. A equação da curva tem a forma: $\mathrm{P}(\mathrm{h})=113,7422 \operatorname{sen}(2 \pi \mathrm{h})+26,5895 \cos (2 \pi \mathrm{h})+142,5764$, onde h denota a hora do evento.
\end{abstract}

Palavras-chave: ciclo diário de precipitações; horossazonalidade de chuvas; modelo senoidal de chuvas.

\begin{abstract}
THE RAINFALL DAILY CYCLE FOR FIVE MINUTES TIME INTERVAL IN THE CITY OF FORTALEZA, CEARÁ

The purpose of this paper is to study the daily cycle of rainfall over Fortaleza, Ceará State. A 30 year time series of rainfall data at five minutes intervals was used. The data came from rain gauge records obtained at the meteorological station at Universidade Federal do Ceará, located at the "Campus do Pici". To obtain the daily rainfall cycle, non-linear regression analysis was used. We searched for fitting to two types of curves: polynomial and sinusoidal. The fitting to a third degree polynomial resulted in an approximately sinusoidal shape, with a determination coefficient $r^{2}=0.9118$. However, the fitted curve showed discontinuity between the values of the ordinates at the beginning and ending of the daily cycle. We developed a new polynomial curve removing the discontinuity. The obtained curve was markedly sinusoidal, with $r^{2}=0.8798$. Then, sinusoidal functions fitting were done. We tested the simple sine and a sine-cosine association. With the combination of sine and cosine, the best fitting found is a curve with $\mathrm{r}^{2}=0.9113$, considered as the best representation of the diurnal cycle. The curve equation has the form: $\mathrm{P}(\mathrm{h})=113.7422 \operatorname{sen}(2 \pi \mathrm{h})+26.5895 \cos (2 \pi \mathrm{h})+142.5764$, where $\mathrm{h}$ is the event time. Keywords: daily cycle of precipitation, sinusoidal model of rainfall.
\end{abstract}




\section{INTRODUÇÃO}

O conhecimento das precipitações de uma dada localidade em intervalos de curta duração constitui-se em importante informação de interesse da engenharia hidrológica e, também, da sociedade de um modo geral. Uma das principais aplicações da modelagem e simulação de chuvas de curta duração está na avaliação de riscos hidrológicos ocasionados, por exemplo, por evento de chuvas intensas (Arnaud et al., 2007). O grupo de pesquisa do CEMAGREF (National Centre of Agricultural Mechanisation, Agricultural Engineering, Waterland Forests), na França, desenvolveu um programa denominado SHYPRE (SimulatedHYdrographsfor floodPRobabilityEstimation), o qual trata a precipitação pluvial em intervalos de uma hora, como um processo estocástico, isto é, que estuda a evolução temporal da ocorrência de chuvas. Esse tipo de modelagem, que combina processos meteorológicos com processo hidráulicos e hidrológicos, é atual e de grande utilidade para a sociedade.

Bernardara et al. (2007) estudaram a distribuição de chuva em intervalos de uma hora. Segundo os autores, a maioria dos modelos de chuva encontrados na literatura é do tipo de fluxo, isto é, são modelos que representam a variabilidade da intensidade e da quantidade de chuva em um determinado local, em certo período. Os autores desenvolveram um modelo estocástico que permite gerar séries sintéticas de chuvas em intervalos de uma hora.

A caracterização estatística da estrutura das tempestades, em intervalos menores de um ano, foi objeto de pesquisa de Salvadori e De Michelle (2006). Um evento de chuva, tempestade, é caracterizado por um período úmido (W) seguido de um período seco (D). O período úmido é representado por um pulso retangular de chuva de intensidade I. O estudo permitiu caracterizar as funções de probabilidade dos episódios de uma hora. Contudo, o modelo pode ser aplicado a intervalos de cinco minutos.

Chen et al. (1999) estudaram o ciclo de precipitações na ilha de Taiwan. Os autores utilizaram medições de estações automáticas, na escala de uma hora, no período 1993-1996. Eles encontraram que o pico de precipitações ocorre entre 16:00h e 17:00h, cerca de duas a três horas após a ocorrência do pico de fluxo de massas ocasionados pela brisa.

Kousky (1980) estudou a variação diurna da chuva em toda a região do Nordeste do Brasil. Os dados de chuva diária foram registrados as $09 \mathrm{~h} 00 \mathrm{~min}, 15 \mathrm{~h} 00 \mathrm{~min}$ e $21 \mathrm{~h} 00 \mathrm{~min}$, com os valores da precipitação correspondente ao acumulado nos períodos 21:00-09:00, 09:00-15:00 e 15:00-21:00 horas, respectivamente. No que se refere ao estado do Ceará, os resultados mostraram que a precipitação máxima ocorre no período entre 21 e 9 horas, tanto ao longo do litoral, com exceção de uma faixa ao leste, na fronteira com o Rio Grande do Norte, e outra, ao oeste, na fronteira com o Piauí, quanto no sul do estado. Já na região continental, distante 150 a $300 \mathrm{~km}$ do litoral, a máxima precipitação acontece entre 15 e 21 horas.

Teixeira (2008) analisou a frequência de ocorrência de chuvas em Fortaleza, em intervalos de uma hora, nos períodos janeiro a junho e julho a dezembro, e constatou que, para ambos os semestres, a grande maioria dos eventos de chuva acontece no período da noite até inicio da manhã, com maior concentração na madrugada. No período da tarde ocorre uma expressiva redução das chuvas, com gradual elevação no início da noite. Os resultados foram validados com a série de 13 anos (1974-1987) coletada na estação meteorológica convencional da Fundação Cearense de Meteorologia e Recursos Hídricos (FUNCEME).

Com o auxílio de imagens de satélite, o autor explica como o fenômeno das brisas se relaciona com a ocorrência de chuvas em Fortaleza. Para ele, a brisa terrestre, associada à forma geométrica côncava do litoral entre Fortaleza e o norte do Rio Grande do Norte e aos ventos alísios, parece favorecer a ocorrência de chuvas em Fortaleza no período da madrugada e inicio da manhã. Quanto à brisa marinha, esta favorece a ocorrência de chuva mais no interior do continente e explica a baixa ocorrência de chuva em Fortaleza no período da tarde.

Uma pesquisa no tema deve-se a Santos e Silva (2013), que estudou o ciclo diário e semidiário de precipitação na região costeira do Norte do Brasil. O autor utilizou 13 anos de dados ininterruptos de precipitação estimados por meio do algoritmo 3B42-V6 da missão TRMM e o método de análise harmônica para estudar o ciclo quadrimestral de precipitações diárias. $\mathrm{O}$ estudo determinou a amplitude, a fase e a variância explicada pelo harmônico diário. O quadrimestre JFMA foi o que apresentou maior amplitude, ocorrendo no Nordeste do Pará. A amplitude diminui na direção do interior do continente. A precipitação máxima ocorre à noite sobre o oceano. Nas localidades situadas até $100 \mathrm{~km}$ da linha da costa, verifica-se a predominância de chuva máxima no período da noite. Para áreas entre 100 e $350 \mathrm{~km}$ de distância da costa, o máximo de precipitação ocorre entre 14 e 17 horas. Em algumas regiões entre 350 e 450 km a máxima precipitação ocorre no inicio da manhã, como a parte central do Pará.

Assis e Padilha (2002) estudaram o ciclo anual, em escala mensal, da pluviosidade na cidade de Pelotas, RS. A precipitação média mensal foi decomposta em ondas harmônicas, chegando-se à conclusão de que as ondas semestral, quadrimestral e bimestral, juntas, explicam 98\% das precipitações que ocorrem em Pelotas.

No Brasil, a modelagem para previsão de tempo obteve grandes conquistas, em decorrência da introdução da análise numérica para o tempo, por meio dos computadores de alto desempenho do Centro de Previsão de Tempo e Estudos Climáticos (CPTEC). No entanto, para Diniz et al. (2008): 
"tanto o CPTEC como outros centros, utilizam-se de modelos estatísticos para previsões climáticas, porém com dificuldade no que se refere à regionalização e falha em condições não típicas". Os autores estudaram o regime de chuva diário com avaliação da sazonalidade em escala mensal.

O primeiro estudo de horossazonalidade em Fortaleza foi desenvolvido para eventos relacionados a anos de contrastes climáticos, La Niña e El Niño (Lima, 2005); (Lima et al., 2006). Os trabalhos apresentaram curvas empíricas para diversas situações, porém não avançaram para a análise de regressão não linear, como abordada na presente pesquisa.

O objetivo deste artigo é obter uma função que modele o ciclo da precipitação pluvial diária do município de Fortaleza em acumulados de cinco minutos. Para ajustar curvas periódicas ou não ao ciclo, utilizou-se a análise de regressão não linear. $\mathrm{O}$ artigo está organizado em quatro seções. $\mathrm{Na}$ introdução, apresentam-se a descrição do problema e a revisão de literatura. Na seção 2, são descritos a área de estudo, os dados e os métodos adotados. A seção 3 é dedicada à aplicação dos modelos desenvolvidos na seção 2 e à apresentação dos resultados. Conclusões e recomendações para trabalhos futuros são apresentados na seção 4.

\section{DADOS E MÉTODOS}

\subsection{Descrição da área}

O município de Fortaleza, capital do Estado do Ceará, situa-se a $3^{\circ} 47^{\prime} 25^{\prime \prime}$ 'S de latitude e $38^{\circ} 30^{\prime} 29,9^{\prime \prime} \mathrm{W}$ de longitude (Figura 1). De frente para o mar do Atlântico, Fortaleza tem 34 $\mathrm{km}$ de praias, altitude média de 21 metros e área de $313,8 \mathrm{~km}^{2}$. A umidade relativa média do ar é $78 \%$.

A temperatura média anual de Fortaleza é de $26^{\circ} \mathrm{C}$, sendo dezembro e janeiro os meses mais quentes e julho o mais frio. As chuvas se concentram entre fevereiro e maio, com média pluviométrica de $1600 \mathrm{~mm}$, sendo o mês de abril o mais chuvoso e novembro o mais seco.

São duas as estações bem definidas no ano: a época chuvosa, localmente chamada de inverno, que se estende de janeiro a julho, e a época seca, de agosto a dezembro, chamada de verão.

Apesar de constatada uma distribuição irregular da precipitação, intra-anos e meses, visualiza-se um comportamento cíclico ou periódico do fenômeno da precipitação, seja na escala anual, na mensal ou na diária.

\subsection{Dados}

Os dados utilizados nesse estudo consistem da chuva registrada no posto pluviométrico da estação meteorológica do

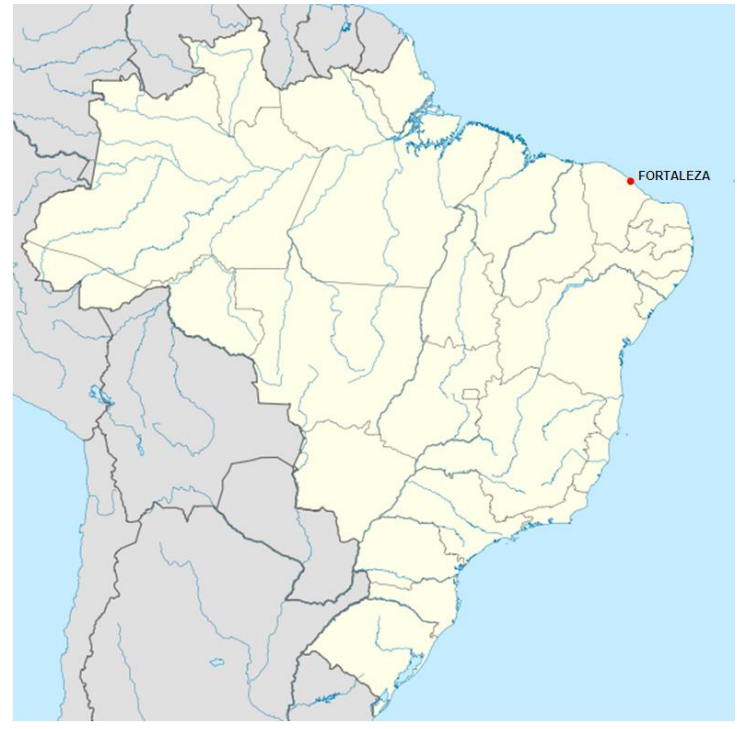

Figura 1 - Mapa de localização da área de estudo Fonte: http://pt.wikipedia.org/wiki/Ficheiro:Brazil_location_map.svg

Centro de Ciências Agrárias da Universidade Federal do Ceará, no Campus do Pici, Fortaleza, CE, durante 30 anos (janeiro de 1970 a dezembro 1999), em intervalos de cinco minutos. A série foi tabulada em 288 classes, com início às 09 h00min e término às $09 \mathrm{~h} 00 \mathrm{~min}$ do dia seguinte.

A chuva observada durante os 5 minutos de uma classe, com início em h:min e término em h:min +5 , é registrada no limite superior da classe, ou seja, em h:min +5 . Assim, os dados da precipitação acumulada em 30 anos, medida em intervalos de 5 minutos, foram estruturados conforme a Tabela 1 .

\subsection{Métodos}

Para o ajustamento dos modelos aos dados observados, utilizou-se a técnica da regressão não linear, de acordo com o método dos mínimos quadrados (MMQ). A medida do ajustamento da função modeladora aos dados é expressa pelo valor do coeficiente de determinação, denotado por $\mathrm{r}^{2}$.

Aos 288 pares $\left(\mathrm{h}_{\mathrm{i}}, \mathrm{P}_{\mathrm{i}}\right)$, correspondentes a hora e ao total da precipitação observada, durante um intervalo de tempo de 5 minutos, são ajustadas funções, as quais se pretende que modele o evento relativo à ocorrência de chuvas no município de Fortaleza.

Foi analisada a modelagem dos dados por meio de funções polinomial e senoidal. No caso polinomial, é considerado o polinômio do $3^{\circ}$ grau, na sua forma comum, e na forma condicionada, isto é, um polinômio do $3^{\circ}$ grau sujeito a uma condição especificada. Tais modelos são referenciados com Polinomial Acíclico e Polinomial Cíclico, respectivamente. 
Tabela 1 - Organização das Precipitações Observadas

\begin{tabular}{|c|c|c|c|c|}
\hline \multirow{2}{*}{ Classe } & \multirow{2}{*}{ Intervalo: $\left(\mathbf{h}_{\mathbf{i}}, \mathbf{h}_{\mathbf{i}+5 \min }\right]$} & \multicolumn{3}{|c|}{ Precipitação Registrada (mm) } \\
\hline & & Hora de Registro & Total 30 Anos & Média Anual \\
\hline 1 & (09h00min, 09h05min] & 09h05min & 243,70 & 8,12 \\
\hline 2 & (09h05min, 09h10min] & 09h10min & 249,90 & 8,33 \\
\hline 3 & (09h10min, 09h15min] & $09 \mathrm{~h} 15 \mathrm{~min}$ & 184,00 & 6,13 \\
\hline 4 & (09h15min, 09h20min] & 09h20min & 259,50 & 8,33 \\
\hline 5 & (09h20min, 09h25min] & $09 \mathrm{~h} 25 \mathrm{~min}$ & 246,30 & 6,13 \\
\hline (...... & n.......................... & .................. & .... & ....... \\
\hline ....... & 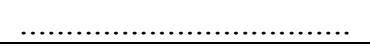 & ................ & .............. & $\ldots \ldots$. \\
\hline 286 & (08h45min, 08h50min] & $08 \mathrm{~h} 50 \mathrm{~min}$ & 162,40 & 5,41 \\
\hline 287 & (08h50min, 08h55min] & $08 \mathrm{~h} 55 \mathrm{~min}$ & 134,60 & 4,49 \\
\hline 288 & (08h55min, 09h00min] & 09h00min & 125,30 & 4,18 \\
\hline
\end{tabular}

Dados do Pluviógrafo do Centro de Ciências Agrárias/UFC (Período:1970 - 1999)

No tocante ao modelo senoidal, é considerada uma função envolvendo apenas o seno, e outra, contemplando uma combinação de senos e cossenos. Tais modelos são referenciados por Senoidal I e II, respectivamente.

\subsubsection{Modelo polinomial acíclico}

Com esse modelo, busca-se um polinômio do $3^{\circ}$ grau, $\mathrm{P}(\mathrm{h})$, que ajusta o total das precipitações diárias, acumulada em intervalos de cinco minutos, durante um período de 30 anos (1970-1999).

Seja o polinômio $\mathrm{P}(\mathrm{h})=\mathrm{Ah}^{3}+\mathrm{Bh}^{2}+\mathrm{Ch}+\mathrm{D}$.

Os coeficientes A, B, C e D de $\mathrm{P}(\mathrm{h})$ são determinados pelo MMQ (Storch e Zwiers, 1999); (Press et al., 1989).

$$
\left(\begin{array}{cccc}
\sum_{i=1}^{n} h^{3} & \sum_{i=1}^{n} h^{2} & \sum_{i=1}^{n} h & n \\
\sum_{i=1}^{n} h^{4} & \sum_{i=1}^{n} h^{3} & \sum_{i=1}^{n} h^{2} & \sum_{i=1}^{n} h \\
\sum_{i=1}^{n} h^{5} & \sum_{i=1}^{n} h^{4} & \sum_{i=1}^{n} h^{3} & \sum_{i=1}^{n} h^{2} \\
\sum_{i=1}^{n} h^{6} & \sum_{i=1}^{n} h^{5} & \sum_{i=1}^{n} h^{4} & \sum_{i=1}^{n} h^{3}
\end{array}\right) x\left(\begin{array}{l}
A \\
B \\
C \\
D
\end{array}\right)=\left(\begin{array}{c}
\sum_{i=1}^{n} P(h) \\
\sum_{i=1}^{n} P(h) \cdot h \\
\sum_{i=1}^{n} P(h) \cdot h^{2} \\
\sum_{i=1}^{n} P(h) \cdot h^{3}
\end{array}\right)
$$

$\mathrm{P}(\mathrm{h})$ é um polinômio comum. O termo acíclico utilizado visa tão somente diferenciar os polinômios considerados nessa seção daqueles da próxima seção, os quais são obtidos ao se impor ao polinômio (Equação 1) uma condição que o torna cíclico, no sentido de que seus valores se igualam nos pontos extremos do intervalo de um dia.

\subsubsection{Modelo polinomial cíclico}

O Modelo Polinomial Acíclico apresenta o inconveniente de estimar valores diferentes nos extremos do intervalo de um dia, isto é, $\mathrm{P}\left(\mathrm{h}_{1}\right) \neq \mathrm{P}\left(\mathrm{h}_{1}+24 \mathrm{~h}\right)$. Essa descontinuidade contraria a constatação da natureza cíclica do fenômeno estudado. Para contornar esse problema, é inserido no polinômio (Equação 1) a condição $\mathrm{P}\left(\mathrm{h}_{1}\right)=\mathrm{P}\left(\mathrm{h}_{1}+24 \mathrm{~h}\right)$, impondo, desse modo, a continuidade do fenômeno no intervalo de um dia. O polinômio assim obtido, conforme mostrado a seguir, é referenciado por Polinômio Cíclico.

Será considerada uma função polinomial do $3^{\circ}$ grau, $\mathrm{P}(\mathrm{h})$, sujeita à condição $\mathrm{P}\left(\mathrm{h}_{2}\right)=\mathrm{P}\left(\mathrm{h}_{1}\right)$, com $\mathrm{h}_{2}=\mathrm{h}_{1}+24 \mathrm{~h}$.

Seja $\mathrm{P}(\mathrm{h})=\mathrm{Ah}^{3}+\mathrm{Bh}^{2}+\mathrm{Ch}+\mathrm{D}$

Assim,

$$
\begin{aligned}
& \mathrm{P}\left(\mathrm{h}_{1}\right)=\mathrm{Ah}_{1}^{3}+\mathrm{Bh}_{1}^{2}+\mathrm{Ch}_{1}+\mathrm{D} \\
& \mathrm{P}\left(\mathrm{h}_{2}\right)=\mathrm{Ah}_{2}^{3}+\mathrm{Bh}_{2}^{2}+\mathrm{Ch}_{2}+\mathrm{D}
\end{aligned}
$$

Sob a condição $\mathrm{P}\left(\mathrm{h}_{2}\right)=\mathrm{P}\left(\mathrm{h}_{1}\right)$, tem-se:

$$
\begin{aligned}
& \mathrm{Ah}_{2}^{3}+\mathrm{Bh}_{2}^{2}+\mathrm{Ch}_{2}+\mathrm{D}=\mathrm{Ah}_{1}^{3}+\mathrm{Bh}_{1}^{2}+\mathrm{Ch}_{1}+\mathrm{D} \\
& \mathrm{A}\left[\mathrm{h}_{2}^{3}-\mathrm{h}_{1}^{3}\right]+\mathrm{B}\left[\mathrm{h}_{2}^{2}-\mathrm{h}_{1}^{2}\right]=\mathrm{C}\left[\mathrm{h}_{1}-\mathrm{h}_{2}\right] \\
& \text { Portanto, } \mathrm{C}=\frac{\mathrm{A}\left[\mathrm{h}_{2}^{3}-\mathrm{h}_{1}^{3}\right]}{\left[\mathrm{h}_{1}-\mathrm{h}_{2}\right]}+\frac{\mathrm{B}\left[\mathrm{h}_{2}^{2}-\mathrm{h}_{1}^{2}\right]}{\left[\mathrm{h}_{1}-\mathrm{h}_{2}\right]} \\
& \text { Fazendo } \alpha=\frac{\left[\mathrm{h}_{2}^{3}-\mathrm{h}_{1}^{3}\right]}{\left[\mathrm{h}_{1}-\mathrm{h}_{2}\right]} \text { e } \beta=\frac{\left[\mathrm{h}_{2}^{2}-\mathrm{h}_{1}^{2}\right]}{\left[\mathrm{h}_{1}-\mathrm{h}_{2}\right]}, \text { tem-se } \\
& C=\alpha A+\beta B
\end{aligned}
$$

Inserindo C na Equação 1, obtém-se:

$$
P(h)=A h^{3}+B h^{2}+(\alpha A+\beta B) h+D
$$

Portanto, $\mathrm{P}(\mathrm{h})=\mathrm{A}\left[\mathrm{h}^{3}+\alpha \mathrm{h}\right]+\mathrm{B}\left[\mathrm{h}^{2}+\beta \mathrm{h}\right]+\mathrm{D}$

Fazendo $\mathrm{x}=\left(\mathrm{h}^{3}+\alpha \mathrm{h}\right)$ e $\mathrm{y}=\left(\mathrm{h}^{2}+\beta \mathrm{h}\right)$, tem-se:

$$
\mathrm{P}(\mathrm{h})=\mathrm{Ax}+\mathrm{By}+\mathrm{D}
$$

A aplicação do MMQ à função da Equação 2 define o sistema a seguir, que permite determinar as constantes $\mathrm{A}, \mathrm{B}$ e 
D que minimizam a soma dos quadrados dos erros.

$$
\left(\begin{array}{lll}
\sum_{\mathrm{i}=1}^{\mathrm{n}} x_{i} & \sum_{\mathrm{i}=1}^{\mathrm{n}} y_{i} & \mathrm{n} \\
\sum_{\mathrm{i}=1}^{\mathrm{n}} x_{i}^{2} & \sum_{\mathrm{i}=1}^{\mathrm{n}} x_{i} y_{i} & \sum_{\mathrm{i}=1}^{\mathrm{n}} x_{i} \\
\sum_{\mathrm{i}=1}^{\mathrm{n}} x_{i} y_{i} & \sum_{\mathrm{i}=1}^{\mathrm{n}} y_{i}^{2} & \sum_{\mathrm{i}=1}^{\mathrm{n}} y_{i}
\end{array}\right) x\left(\begin{array}{l}
A \\
B \\
D
\end{array}\right)=\left(\begin{array}{l}
\sum_{i=1}^{n} P\left(h_{i}\right) \\
\sum_{i=1}^{n} P\left(h_{i}\right) x_{i} \\
\sum_{i=1}^{n} P\left(h_{i}\right) y_{i}
\end{array}\right)
$$

Uma vez conhecidos $\mathrm{A}, \mathrm{B}$ e $\mathrm{D}$, determina-se $\mathrm{C}=\alpha \mathrm{A}+$ $\beta \mathrm{B}$, e assim o Polinômio Cíclico do $3^{\circ} \mathrm{Grau}$ :

$$
\mathrm{P}(\mathrm{h})=\mathrm{Ah}^{3}+\mathrm{Bh}^{2}+\mathrm{Ch}+\mathrm{D} \text {. }
$$

\subsubsection{Modelo senoidal I}

Dados $\mathrm{n}$ pares $\left(\mathrm{X}_{\mathrm{i}}, \mathrm{Y}_{\mathrm{i}}\right)$ de valores, sendo $\mathrm{X}$ a hora e $\mathrm{Y}$ a precipitação observada, deseja-se ajustar uma função simples, da forma $S\left(X_{i}\right)=A \cdot \operatorname{sen}\left(2 \pi X_{i}\right)+C$, com A e C constantes a serem determinadas.

Assim, o valor estimado de $\mathrm{S}_{\mathrm{i}}$, correspondente a $\mathrm{X}_{\mathrm{i}}$, é dado por $\mathrm{S}_{\mathrm{i}}=\mathrm{A} \cdot \operatorname{sen}\left(2 \pi \mathrm{X}_{\mathrm{i}}\right)+\mathrm{C}$, sendo o erro $\varepsilon_{\mathrm{i}}=\left(\mathrm{Y}_{\mathrm{i}}-\mathrm{S}_{\mathrm{i}}\right)=$ $\mathrm{Y}_{\mathrm{i}}-\mathrm{A} \cdot \operatorname{sen}\left(2 \pi \mathrm{X}_{\mathrm{i}}\right)-\mathrm{C}$.

Seja $\mathrm{Z}$ a soma dos quadrados dos erros:

$$
Z=\sum_{1}^{n} \varepsilon_{i}^{2}=\sum_{1}^{n}\left[Y_{i}-A \cdot \operatorname{sen}\left(2 \pi X_{i}\right)-C\right]^{2}
$$

Pelo MMQ, as constantes A e C são determinadas ao se impor a condição de que a soma dos quadrados dos erros seja mínima, isto é: $\partial \mathrm{Z} / \partial \mathrm{C}=\partial \mathrm{Z} / \partial \mathrm{A}=0$

$$
\begin{aligned}
& \frac{\partial Z}{\partial C}=\sum_{1}^{n} 2\left[Y_{i}-A \cdot \operatorname{sen}\left(2 \pi X_{i}\right)-C\right](-1)=0 \\
& \frac{\partial Z}{\partial A}=\sum_{1}^{n} 2\left[Y_{i}-A \cdot \operatorname{sen}\left(2 \pi X_{i}\right)-C\right] \cdot\left[-\operatorname{sen}\left(2 \pi X_{i}\right)\right]=0
\end{aligned}
$$

A solução analítica para o sistema formado pelas Equações 4 e 5, é dada pelas Equações 6 e 7:

$$
\begin{aligned}
& \mathrm{A}=\frac{\overline{\mathrm{Y}_{\mathrm{i}} \cdot \operatorname{sen}\left(2 \pi \mathrm{X}_{\mathrm{i}}\right)}-\overline{\mathrm{Y}_{\mathrm{i}}} \cdot \overline{\operatorname{sen}\left(2 \pi \mathrm{X}_{\mathrm{i}}\right)}}{\overline{\operatorname{sen}^{2}\left(2 \pi \mathrm{X}_{\mathrm{i}}\right)}-\left(\overline{\operatorname{sen}\left(2 \pi \mathrm{X}_{\mathrm{i}}\right)}\right)^{2}} \\
& C=\overline{Y_{i}}-\overline{A \operatorname{sen}\left(2 \pi X_{i}\right)}
\end{aligned}
$$

(Notação: $\overline{\mathrm{W}}$ indica a média de $\mathrm{W}$ )

\subsubsection{Modelo senoidal II}

Como alternativa ao modelo senoidal I, buscou-se uma função formada pela combinação de senos e cossenos, da forma $\mathrm{S}(\mathrm{h})=\mathrm{A} \cdot \operatorname{sen}(2 \pi \mathrm{h})+\mathrm{B} \cdot \cos (2 \pi \mathrm{h})+\mathrm{C}$.

Uma vez conhecidos $n$ pares $\left(\mathrm{X}_{\mathrm{i}}, \mathrm{Y}_{\mathrm{i}}\right)$ de valores $(\mathrm{X}=$ hora e $\mathrm{Y}=$ precipitação), quer-se ajustar aos dados uma função $\mathrm{S}\left(\mathrm{X}_{\mathrm{i}}\right)$ $=\mathrm{A} \cdot \operatorname{sen}\left(2 \pi \mathrm{X}_{\mathrm{i}}\right)+\mathrm{B} \cdot \cos \left(2 \pi \mathrm{X}_{\mathrm{i}}\right)+\mathrm{C}$, sendo $\mathrm{A}, \mathrm{B}$ e $\mathrm{C}$ constantes a serem determinadas.
O valor estimado $\left(\mathrm{S}_{\mathrm{i}}\right)$, o erro $\left(\varepsilon_{\mathrm{i}}\right)$ e a soma dos quadrados dos erros (Z) são dados por:

$$
\begin{aligned}
& S_{i}=A \cdot \operatorname{sen}\left(2 \pi X_{i}\right)+B \cos \left(2 \pi X_{i}\right)+C \\
& \varepsilon_{i}=\left(Y_{i}-S_{i}\right)=Y_{i}-A \cdot \operatorname{sen}\left(2 \pi X_{i}\right)-B \cdot \cos \left(2 \pi X_{i}\right)-C \\
& \mathrm{Z}=\sum_{1}^{\mathrm{n}} \varepsilon_{\mathrm{i}}^{2}=\sum_{1}^{\mathrm{n}}\left[\mathrm{Y}_{\mathrm{i}}-\mathrm{A} \cdot \operatorname{sen}\left(2 \pi \mathrm{X}_{\mathrm{i}}\right)-\mathrm{B} \cdot \cos \left(2 \pi \mathrm{X}_{\mathrm{i}}\right)-\mathrm{C}\right]^{2}
\end{aligned}
$$

As constantes A, B e C são determinas pelo MMQ, com a solução do sistema da Equação 11, formado a partir das Equações 8, 9 e 10.

$$
\begin{aligned}
& \frac{\partial Z}{\partial C}=\sum_{i=1}^{n} 2\left[Y_{i}-A \cdot \operatorname{sen}\left(2 \pi X_{i}\right)-B \cos \left(2 \pi X_{i}\right)-C\right](-1)=0 \\
& \frac{\partial Z}{\partial B}=\sum_{i=1}^{n} 2\left[Y_{i}-A \operatorname{sen}\left(2 \pi X_{i}\right)-B \cos \left(2 \pi X_{i}\right)-B\right]\left(-\cos \left(2 \pi X_{i}\right)\right)=0
\end{aligned}
$$

$$
\frac{\partial Z}{\partial A}=\sum_{i=1}^{n} 2\left[Y_{i}-A \operatorname{sen}\left(2 \pi X_{i}\right)-B \cos \left(2 \pi X_{i}\right)-C\right]\left(-\operatorname{sen}\left(2 \pi X_{i}\right)\right)=0
$$

Desse modo, A, B e C são determinadas ao se resolver o seguinte sistema:

$$
\begin{aligned}
& \left(\begin{array}{lll}
\sum_{\mathrm{i}=1}^{\mathrm{n}} \operatorname{sen}\left(2 \pi \mathrm{X}_{\mathrm{i}}\right) & \sum_{\mathrm{i}=1}^{\mathrm{n}} \cos \left(2 \pi \mathrm{X}_{\mathrm{i}}\right) & \mathrm{n} \\
\sum_{\mathrm{i}=1}^{\mathrm{n}} \operatorname{sen}\left(2 \pi \mathrm{X}_{\mathrm{i}}\right) \cos \left(2 \pi \mathrm{X}_{\mathrm{i}}\right) & \sum_{\mathrm{i}=1}^{\mathrm{n}} \cos \left(2 \pi \mathrm{X}_{\mathrm{i}}\right) \cos \left(2 \pi \mathrm{X}_{\mathrm{i}}\right) & \sum_{\mathrm{i}=1}^{\mathrm{n}} \cos \left(2 \pi \mathrm{X}_{\mathrm{i}}\right) \\
\sum_{\mathrm{i}=1}^{\mathrm{n}} \operatorname{sen}\left(2 \pi \mathrm{X}_{\mathrm{i}}\right) \operatorname{sen}\left(2 \pi \mathrm{X}_{\mathrm{i}}\right) & \sum_{\mathrm{i}=1}^{\mathrm{n}} \cos \left(2 \pi \mathrm{X}_{\mathrm{i}}\right) \operatorname{sen}\left(2 \pi \mathrm{X}_{\mathrm{i}}\right) & \sum_{\mathrm{i}=1}^{\mathrm{n}} \operatorname{sen}\left(2 \pi \mathrm{X}_{\mathrm{i}}\right)
\end{array}\right) \\
& \times\left(\begin{array}{l}
A \\
B \\
C
\end{array}\right)=\left(\begin{array}{l}
\sum_{i=1}^{n} Y_{i} \\
\sum_{i=1}^{n} Y_{i} \cos \left(2 \pi X_{i}\right) \\
\sum_{i=1}^{n} Y_{i} \operatorname{sen}\left(2 \pi X_{i}\right)
\end{array}\right)
\end{aligned}
$$

\subsubsection{Modelo composto por polinômio e senoide}

Por fim, pesquisou-se um modelo composto de polinômio e senoide. No caso foi considerada a função $F(h)$, formada pela soma do Polinômio Cíclico e a Senoide II.

$$
F(h)=A_{1}\left[h^{3}+\alpha h\right]+B_{1}\left[h^{2}+\beta h\right]+A_{2} \operatorname{sen}(2 \pi h)+B_{2} \cos (2 \pi h)+E
$$

Os coeficientes $A_{1}, B_{1}, A_{2}, B_{2}$ e $E$ são determinados ao se resolver o sistema a seguir, sendo $x=h^{3}-\alpha h, y=h^{2}-\beta h$, $\mathrm{z}=\operatorname{sen}(2 \pi \mathrm{h}), \mathrm{e} \mathrm{w}=\cos (2 \pi \mathrm{h})$ :

$$
\left(\begin{array}{lllll}
\sum_{i=1}^{n} x_{i} & \sum_{i=1}^{n} y_{i} & \sum_{i=1}^{n} z_{i} & \sum_{i=1}^{n} w_{i} & n \\
\sum_{i=1}^{n} x_{i} \cdot x_{i} & \sum_{i=1}^{n} x_{i} \cdot y_{i} & \sum_{i=1}^{n} x_{i} \cdot z_{i} & \sum_{i=1}^{n} x_{i} \cdot w_{i} & \sum_{i=1}^{n} x_{i} \\
\sum_{i=1}^{n} y_{i} \cdot x_{i} & \sum_{i=1}^{n} y_{i} \cdot y_{i} & \sum_{i=1}^{n} y_{i} \cdot z_{i} & \sum_{i=1}^{n} y_{i} \cdot w_{i} & \sum_{i=1}^{n} y_{i} \\
\sum_{i=1}^{n} z_{i} \cdot x_{i} & \sum_{i=1}^{n} z_{i} \cdot y_{i} & \sum_{i=1}^{n} z_{i} \cdot z_{i} & \sum_{i=1}^{n} z_{i} \cdot w_{i} & \sum_{i=1}^{n} z_{i} \\
\sum_{i=1}^{n} w_{i} \cdot x_{i} & \sum_{i=1}^{n} w_{i} \cdot y_{i} & \sum_{i=1}^{n} w_{i} \cdot z_{i} & \sum_{i=1}^{n} w_{i} \cdot w_{i} & \sum_{i=1}^{n} w_{i}
\end{array}\right) x\left(\begin{array}{l}
A_{1} \\
B_{1} \\
A_{2} \\
B_{2} \\
E
\end{array}\right)=\left(\begin{array}{l}
\sum_{i=1}^{n} P\left(h_{i}\right) \\
\sum_{i=1}^{n} P\left(h_{i}\right) \cdot x_{i} \\
\sum_{i=1}^{n} P\left(h_{i}\right) \cdot y_{i} \\
\sum_{i=1}^{n} P\left(h_{i}\right) \cdot z_{i} \\
\sum_{i=1}^{n} P\left(h_{i}\right) \cdot w_{i}
\end{array}\right)
$$




\section{RESULTADOS E DISCUSSÃO}

Utilizando-se os dados de precipitação registrados no posto pluviométrico da estação meteorológica da Universidade Federal do Ceará, no Campus do Pici, Fortaleza, CE, em intervalos de cinco minutos, e totalizados no período de 30 anos, foram determinadas as curvas que modelam o ciclo diário da precipitação no município de Fortaleza.

\subsection{Modelo polinomial acíclico}

Para esse modelo, o ajustamento dos dados diários determina o polinômio do $3^{\circ}$ grau a seguir, o qual tem coeficiente de determinação $r^{2}=0,9118$ :

$P(h)=-2718,7703 h^{3}+7707,3141^{2}-6665,2231 h+1847,7283$.

Os coeficientes do polinômio foram obtidos diretamente a partir dos dados por meio do uso da opção Adicionar Linha de Tendência do Microsoft Office Home and Student 2007.

Os valores de pico mínimo e máximo $\left(\mathrm{P}^{\prime}(\mathrm{h})=0\right)$ da precipitação estimada por $\mathrm{P}(\mathrm{h})$ ocorrem às $16 \mathrm{~h} 05(24,14 \mathrm{~mm}) \mathrm{e}$ $5 \mathrm{~h} 17$ (250,84mm), respectivamente, com intervalo de 13 horas e 12 minutos entre as ocorrências (Figura 2).

$\mathrm{P}(\mathrm{h})=-2718,7703 \mathrm{~h}^{3}+7707,3141 \mathrm{~h}^{2}-6665,2231 \mathrm{~h}+$ $1847,7283\left(\mathrm{r}^{2}=0,9118\right)$.

Como é possível observar, apesar do excelente coeficiente de determinação, o polinômio em questão tem o inconveniente de estimar o valor da precipitação em $\mathrm{h}_{1}=09 \mathrm{~h} 05$ muito diferente daquele estimado para $\mathrm{h}_{2}=\mathrm{h}_{1}+24$ horas, qual seja, 09h05 do dia seguinte, evidenciando a descontinuidade dos valores estimados nos pontos nos extremos do intervalo de um dia.
$\mathrm{P}\left(\mathrm{h}_{1}\right)=281,74 \mathrm{~mm} ; \mathrm{P}\left(\mathrm{h}_{2}\right)=\mathrm{P}\left(\mathrm{h}_{1}+24 \mathrm{~h}\right)=183,81 \mathrm{~mm} ;$

$\mathrm{P}\left(\mathrm{h}_{1}\right)-\mathrm{P}\left(\mathrm{h}_{2}\right)=97,93 \mathrm{~mm}$ (descontinuidade).

\subsection{Modelo polinomial cíclico}

A aplicação da metodologia descrita em 2.3.2 possibilita a obtenção do polinômio cíclico que ajusta os dados das precipitações observadas, sujeito à condição

$\mathrm{P}\left(\mathrm{h}_{1}\right)=\mathrm{P}\left(\mathrm{h}_{1}+24 \mathrm{~h}\right)$.

Assim,

$\mathrm{h}_{1}=9: 05 \mathrm{~h} \mathrm{e} \mathrm{h}_{2}=\mathrm{h}_{1}+24 \mathrm{~h}$,

$\alpha=-2,5651403$ e $\beta=-1,7569444$,

$\mathrm{x}=\left(\mathrm{h}^{3}-2,5651403 \mathrm{~h}\right)$ e $\mathrm{y}=\left(\mathrm{h}^{2}-1,7569444 \mathrm{~h}\right)$.

$\mathrm{C}=-2,5651403 * \mathrm{~A}-1,7569444 * \mathrm{~B}=5160,6963$.

$\mathrm{P}(\mathrm{h})=\mathrm{A}\left[\mathrm{h}^{3}-2,5651403 \mathrm{~h}\right]+\mathrm{B}\left[\mathrm{h}^{2}-1,7569444 \mathrm{~h}\right]+\mathrm{D}$

Os coeficientes $\mathrm{A}=-2033,3162, \mathrm{~B}=5905,9567$ e $\mathrm{D}=$ 1451,2310, são obtidos a partir de Equação 3. Assim,

$$
\mathrm{C}=-2,5651403 * \mathrm{~A}-1,7569444 * \mathrm{~B}=-5160,6963 .
$$

O Polinômio pesquisado é

$\mathrm{P}(\mathrm{h})=-2033,3162 \mathrm{~h}^{3}+5905,9567 \mathrm{~h}^{2}-5160,6963 \mathrm{~h}+$ 1451,2310 , com $\mathrm{r} 2=0,8798$ e $\mathrm{P}\left(\mathrm{h}_{1}\right)=\mathrm{P}\left(\mathrm{h}_{2}\right)$ : (Figura 3 )

Os valores de pico mínimo e máximo da precipitação estimada por $\mathrm{P}(\mathrm{h})$ ocorrem às $15 \mathrm{~h} 58(33,17 \mathrm{~mm})$ e $6 \mathrm{~h} 29 \mathrm{~m}$ $(257,85 \mathrm{~mm})$, respectivamente, com um intervalo de 14 horas e 31 minutos entre as ocorrências.

$\mathrm{P}(\mathrm{h})=-2033,3162 \mathrm{~h}^{3}+5905,9567 \mathrm{~h}^{2}-5160,6963 \mathrm{~h}+$ 1451,2310 (r2=0,8798).

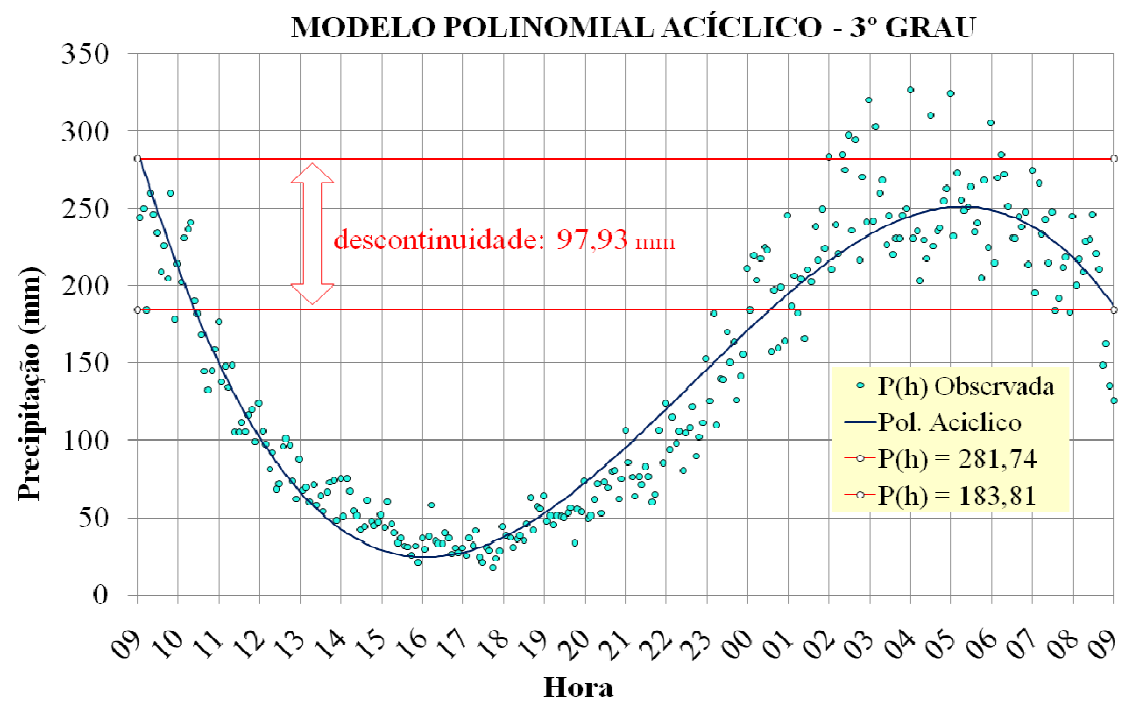

Figura 2 - Polinômio acíclico do $3^{\circ}$ grau 


\subsection{Modelo senoidal I}

Os coeficientes do Modelo Senoidal I são diretamente obtidos das Equações 6 e 7. A senoide determinada, com $\mathrm{r}^{2}=0,8641$ é a seguinte (Figura 4$): \mathrm{S}(\mathrm{h})=113,7422 * \operatorname{sen}(2 \pi \mathrm{h})$ $+142,5764$.

Os valores de pico máximo e mínimo da precipitação estimada por $\mathrm{S}(\mathrm{h})$ ocorrem às $06 \mathrm{~h} 00 \mathrm{~min}(256,32 \mathrm{~mm})$ e 18 h00 min $(28,83 \mathrm{~mm})$, respectivamente, com intervalo de 12 horas entre os picos de mínimo e máximo.

A função $\mathrm{S}(\mathrm{h})$ tem o predicado de ser muito simples, além da vantagem de reproduzir naturalmente a característica cíclica do fenômeno estudado, conforme pode ser visto na Figura 4. Sua desvantagem, em relação aos modelos polinomiais, está no fato de seu coeficiente de determinação $\left(r^{2}=0,8641\right)$ ser inferior ao dos modelos polinomial cíclico e acíclico.

$$
\mathrm{S}(\mathrm{h})=113,7423 * \operatorname{sen}(2 \pi \mathrm{h})+142,5764 \quad(\mathrm{r} 2=0,8641)
$$

\subsection{Modelo senoidal II}

Os coeficientes A, B e C do modelo são determinados através da Equação 11: $\mathrm{A}=113,7422, \mathrm{~B}=26,5895$ e $\mathrm{C}=$ 142,5764, com $\mathrm{r}^{2}=0,9113$. A Figura 5 mostra o gráfico da senoide obtida:

$\mathrm{S}(\mathrm{h})=113,7422 * \operatorname{sen}(2 \pi \mathrm{h})+26,5895 \cos (2 \pi \mathrm{h})+142,5764$ $\left(r^{2}=0,9113\right)$

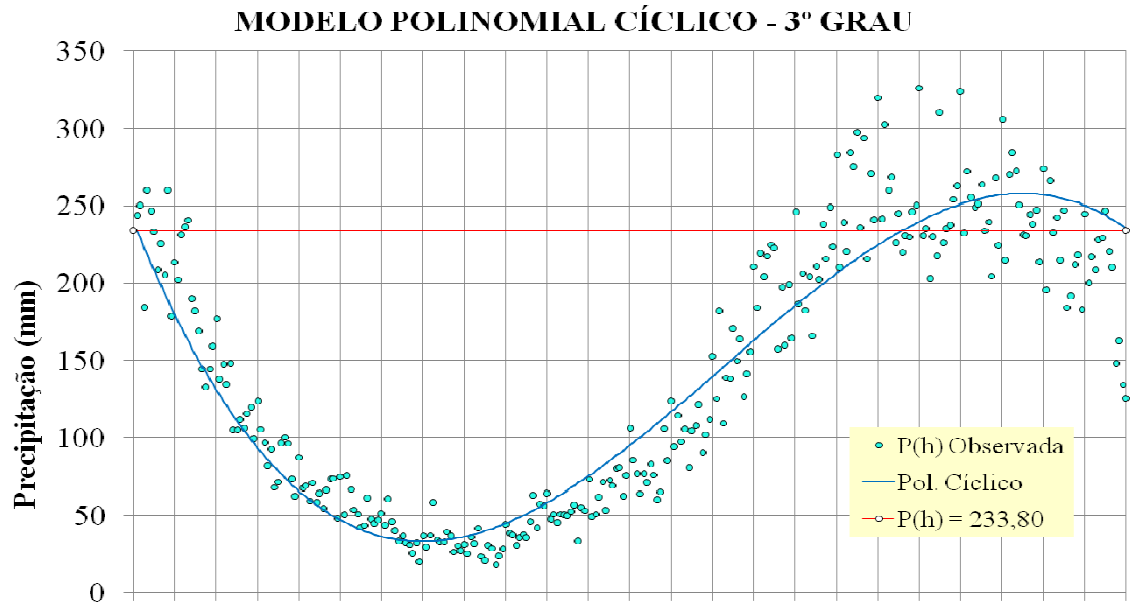

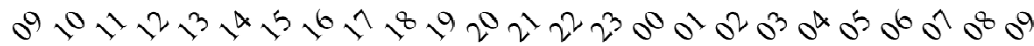

IIora

Figura 3 - Polinômio cíclico do $3^{\circ}$ grau

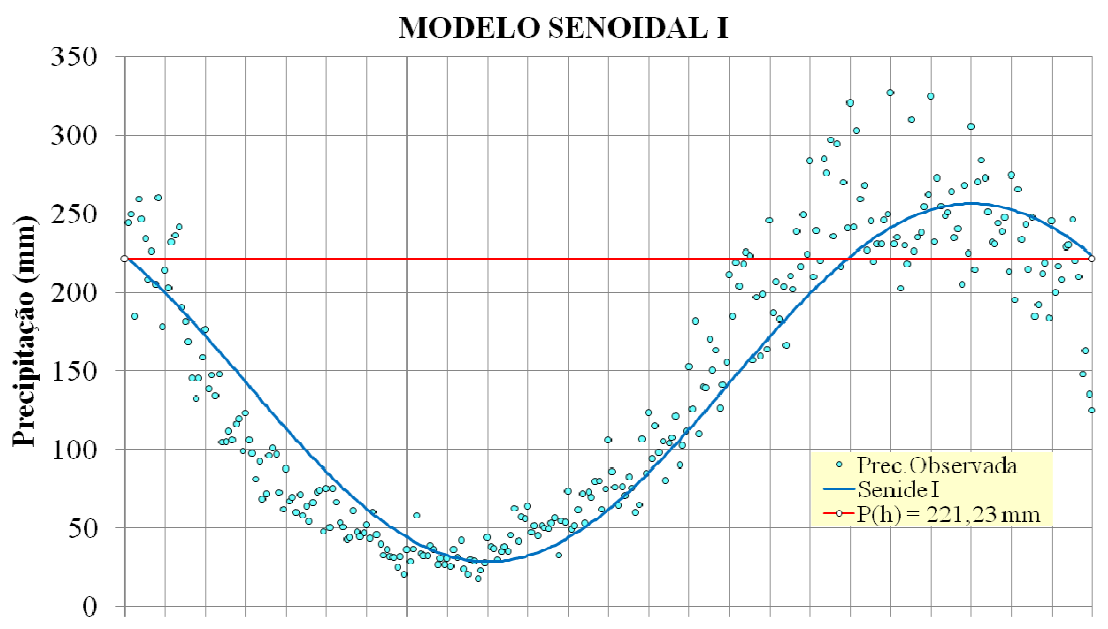

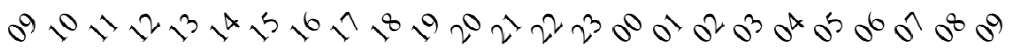

Hora

Figura 4 - Modelo da senoidal I 
Os valores pico máximo e mínimo da precipitação estimada por $\mathrm{S}(\mathrm{h})$ ocorrem às $05 \mathrm{~h} 05 \mathrm{~min}(259,38 \mathrm{~mm})$ e $17 \mathrm{~h} 05 \mathrm{~mm}(25,77 \mathrm{~mm})$, respectivamente, com um intervalo de $12 \mathrm{~h} 00 \mathrm{~min}$ entre as ocorrências.

O modelo Senoidal II reproduz a característica cíclica do fenômeno estudado, tem o coeficiente de determinação $\left(\mathrm{r}^{2}=0,9113\right)$ superior ao dos modelos Senoidal I $\left(\mathrm{r}^{2}=0,8641\right)$ e Polinomial Cíclico $\left(r^{2}=0,8798\right)$, e praticamente igual ao $r^{2}$ do modelo Polinomial Acíclico $\left(\mathrm{r}^{2}=0,9118\right)$.

\subsection{Modelo Composto}

O modelo obtido da soma do Polinômio Cíclico com a Senoidal II mantém a pretendida característica cíclica do modelo pesquisado. Os coeficientes do modelo foram obtidos pelo MMQ. O modelo apresenta um excelente coeficiente de determinação, $\mathrm{r}^{2}=0,9125$, e tem gráfico conforme a Figura 6:

$$
\mathrm{F}(\mathrm{h})=569,5570 \mathrm{~h}^{3}-1431,7199 \mathrm{~h}^{2}+1054,4586 \mathrm{~h}+128,7903 \mathrm{sen}
$$

$(2 \pi \mathrm{h})+50,7155 \cos (2 \pi \mathrm{h})-70,7530 \quad\left(\mathrm{r}^{2}=0,9125\right)$

Os valores de pico máximo e mínimo da precipitação estimada por $\mathrm{F}(\mathrm{h})$ ocorrem às $04 \mathrm{~h} 55 \mathrm{~min}(255,35 \mathrm{~mm})$ e $17 \mathrm{~h}: 05 \mathrm{~min}(22,67 \mathrm{~mm})$, respectivamente, com intervalo de 11h:50min entre os picos de mínimo e máximo.

A Tabela 2 a seguir sumariza as precipitações máximas e mínimas estimadas pelos modelos, a hora de ocorrência e o intervalo entre os picos de mínimo e máximo:

\section{CONCLUSÕES E RECOMENDAÇÕES}

Pelos estudos realizados, pode-se concluir que o modelo polinomial acíclico, apesar de apresentar um

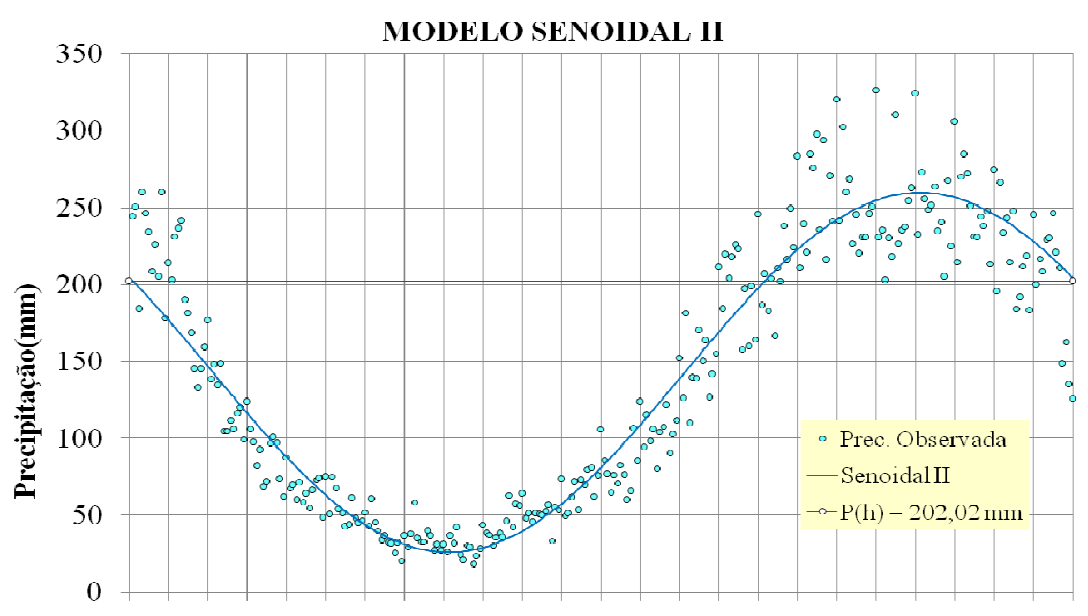

09101112131415161718192021222300010203040506070809

Hora

Figura 5 - Modelo da senoidal I I

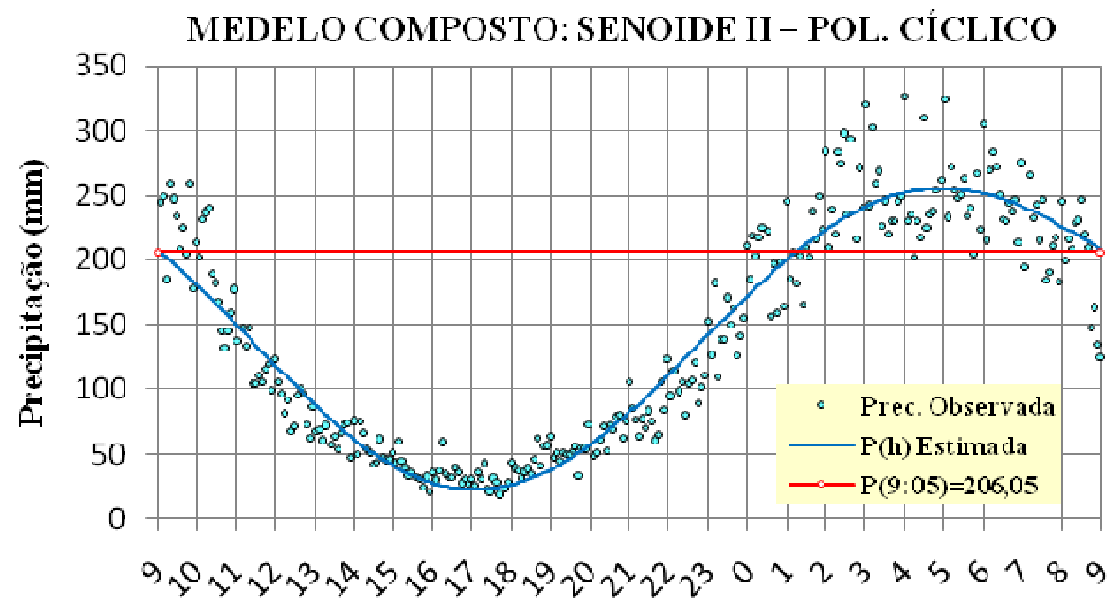

Hora

Figura 6 - Modelo composto: polinômio cíclico + senoide II. 
Tabela 2 - Precipitação máxima x mínima e hora de ocorrência.

\begin{tabular}{|l|c|c|c|c|c|}
\hline \multirow{2}{*}{\multicolumn{1}{|c|}{ MODELO }} & \multicolumn{2}{|c|}{$\begin{array}{c}\text { Precipitação } \\
\text { Mínima }\end{array}$} & \multicolumn{2}{c|}{$\begin{array}{c}\text { Precipitação } \\
\text { Máxima }\end{array}$} & $\begin{array}{c}\text { Ah Entre Picos de } \\
\text { Mínimo e } \\
\end{array}$ \\
\cline { 2 - 5 } & Hora & P(h) & Hora & P(h) & Máximo \\
\hline Pol. Ać́clico & $16: 05$ & 24,14 & $05: 15$ & 250,84 & $13: 10$ \\
\hline Pol. Cíclico & $16: 00$ & 33,17 & $06: 30$ & 257,85 & $14: 30$ \\
\hline Senoidal I & $18: 00$ & 28,83 & $06: 00$ & 256,32 & $12: 00$ \\
\hline Senoidal II & $17: 05$ & 25,77 & $05: 05$ & 259,38 & $12: 00$ \\
\hline Pol. C.+ Senoidal II & $17: 05$ & 22,67 & $04: 55$ & 255,35 & $11: 50$ \\
\hline
\end{tabular}

expressivo coeficiente de determinação $\left(\mathrm{r}^{2}=0,9118\right)$, tem o inconveniente de ser descontínuo nos extremos do intervalo de um dia. Tal descontinuidade pode ser resolvida com o polinômio cíclico, porém ao custo de redução no coeficiente de determinação $\left(r^{2}=0,8798\right)$. Os modelos senoidais apresentam a vantagem de serem naturalmente cíclicos. $\mathrm{O}$ modelo senoidal I é extremamente simples, porém, dentre os modelos estudados, é aquele que apresenta menor coeficiente de determinação $\left(r^{2}=0,8641\right)$. Quanto ao modelo Senoidal II, o coeficiente de determinação $\left(r^{2}=0,9113\right)$ é próximo daquele do modelo polinomial acíclico, sendo igual até a terceira casa decimal.

O modelo composto Polinômio Cíclico+Senoide II é o que apresenta maior coeficiente de determinação $\left(\mathrm{r}^{2}=0,9125\right)$, sendo superior em $0,13 \%$ ao da Senoide II. Contudo, esse modelo tem seis parâmetros, enquanto que o modelo senoidal tem somente três parâmetros. Desse modo, pelo princípio da parcimônia dos parâmetros (Campos, 2009), recomenda-se o modelo da Senoide II como o mais adequado para representar as chuvas em Fortaleza, em intervalos de cinco minutos.

Estudos anteriores sobre a distribuição infradiária de chuvas no Nordeste do Brasil, e em especial sobre o Ceará, concluíram que os eventos de chuvas acontecem de forma crescente durante a noite, madrugada e início da manhã. No decorrer do dia as chuvas se tornam mais escassas, com mínimo no final da tarde e início da noite (Kousky, 1980 e Teixeira, 2008). Os modelos estudados neste artigo validam os resultados referidos, e utilizam formulações matemáticas para obter o ciclo diário e determinar a hora de ocorrência do máximo e do mínimo. Obteve-se, com o modelo senoidal II, a ocorrência do máximo às $5 \mathrm{~h} 05 \mathrm{~m}$ e o mínimo às $17 \mathrm{~h} 05 \mathrm{~m}$.

Essa forma de variação, máximo pela madrugada e mínimo pelo final da tarde, foi explicada por Teixeira (2008) como decorrência dos efeitos das brisas marítimas e terrestres, que atuam no litoral de Fortaleza, durante todo o ano.

Como extensão dessa pesquisa desenvolve-se estudos do fenômeno na escala de tempo mensal. Pretende-se determinar se essas curvas teóricas variam ao longo do ano em decorrência dos sistemas sinóticos que atuam na região, como a Zona de Convergência Intertropical (ZCIT).

\section{REFERÊNCIAS BIBLIOGRÁFICAS}

ARNAUD, P.; FINE, J. A.; LAVABRE, J. An hourly rainfall generation model applicable to all type of climate. Atmospheric Research, v. 85, n.2, p. 230-242, aug. 2007.

ASSIS, S. V. D.; PADILHA, C. K. Periodicidade da precipitação de Pelotas, RS explicada pela análise harmônica. In: Congresso Brasileiro de Meteorologia, 12, 2002, Foz de Iguaçu. Anais... Foz de Iguaçu: SBMET, 2002. p 1-7.

BERNARDARA P.; DE MICHELE, C.; ROSSO, R. A simple model of rain in time: An alternating renewal process of wet and dry states with fractional (non-gaussian) rain intensity. Atmospheric. Research, v. 84, n.4, p. 291-301, jun. 2007. CAMPOS, J. N. B. Lições em modelos e simulação hidrológica, Fortaleza: ASTEF/Expressão Gráfica, 2009.

CHEN,T-C; YEN, M-C; HSIEH, J-C; ARRIT, R. W. Diurnal and seasonal variations of the rainfall measured by the automatic rainfall and meteorological telemetry system in Taiwan. Bulletin of the American Meteorological Society, v. 80, n.11, p.2299-2312, 1999.

DINIZ, G. L.; FONSECA, M.; CAMPELO JR., J. H. Análise harmônica de precipitação em duas localidades da baixada cuiabana. Biomatemática, v. 18, p. 37-48, 2008.

KOUSKY, V. E. Diurnal rainfall variation in the Northeast Brazil. Monthly Weather Review, Boston, v.108, n.4, p. 488-498. 1980.

LIMA, V. P. M. Variabilidade horo-sazonal da precipitação em Fortaleza-Ce, em anos de constrastes climáticos. 2005. 126f. Dissertação (Mestrado em Engenharia Civil). Centro de Tecnologia, Universidade Federal do Ceará, Fortaleza, 2005.

LIMA, V. D. P. M.; CAMPOS, J. N. B.; ALVES, J. M. B. Variabilidade horo-sazonal da precipitação em Fortaleza, Ce em anos de contraste climático.In: Congresso Brasileiro de Meteorologia, 14, 2006, Florianopolis. Anais... Congresso Brasileiro de Meteorologia. Florianóplis: SBMET, p. 1-6, 2006.

SALVADORI, G.; DE MICHELE, C. Statistical characterization of temporal structure of storms. Advances in Water Resources, v. 29, n. 6 p. 827-842. 2006. 
SANTOS E SILVA, C. M. S. E. Ciclo diário e semidiário de precipitação na costa norte do Brasil, Revista Brasileira de Meteorologia, v.28, n.1, 34-42, mar. 2013.

STORCH, H. V.; ZWIERS F. W. Statistical Analysis in Climate Research, New York: Cambridge University PRESS, 1999.

TEIXEIRA, R. F. B. O fenômeno da brisa e sua relação com a chuva sobre Fortaleza-Ce, Revista Brasileira de Meteorologia, v.23, n.3, 282-291, set. 2008.
PRESS, W. H.; FLANNERY, B. P.; TEUKOLSKY, S. A.; et al. Numerical Recipes in FORTRAN, New York: Cambridge University PRESS, 1989. 